\title{
Gantry crane control of a double-pendulum, distributed-mass load, using mechanical wave concepts
}

\author{
W. O'Connor and H. Habibi \\ UCD School of Mechanical and Materials Engineering, UCD Belfield, Dublin 4, Ireland \\ Correspondence to: H. Habibi (hossein.habibi@ucdconnect.ie)
}

Received: 15 November 2012 - Revised: 16 April 2013 - Accepted: 19 April 2013 - Published: 1 July 2013

\begin{abstract}
The overhead trolley of a gantry crane can be moved in two directions in the plane. The trolley is attempting to control the motion of a suspended, rigid-body, distributed mass load, supported by a hook, modelled as a lumped mass, in turn connected to the trolley by a light flexible cable. This flexible system has six degrees of freedom, four variables describing the flexible, hanging load dynamics and two (directly controlled) input variables for the trolley position. The equations of motion are developed and the crane model is verified. Then a form of wave-based control (WBC) is applied to determine what trolley motion should be used to achieve a reference motion of the load, with minimum swing during complex manoeuvres. Despite the trolley's limited control authority over the complex, flexible 3-D dynamics, WBC enables the trolley to achieve very good motion control of the load, in a simple, robust and rapid way, using little sensor information, with all measurements taken at or close to the trolley.
\end{abstract}

\section{Introduction}

In a typical problem of crane control, the challenge is to achieve controlled motion of the load, simultaneously moving it to follow a desired trajectory while actively controlling the swing. The system can be described as under-actuated: only the trolley is directly controlled, and it must indirectly control the swinging load at the far end of the cable. Performance can be measured under various headings, including minimization of sway during motion or on arrival at target, tracking desired motion paths, accurate repositioning payloads in target within the shortest possible time, maximum repetition rate, and safety (Abdel-Rahman et al., 2003). Even with no external disturbances, the trolley motion can cause significant payload pendulation, especially when the dynamics are more complex. Also cranes are inherently lightly damped (Todd et al., 1997). Finally, the load can change significantly, in ways which the controller may not know in advance. All these factors add to the control challenge.

Experienced crane drivers can acquire considerable skill, both in steering a load along a desired trajectory and in damping the swinging, especially on arrival at target. The trajectory might require guiding a heavy load safely around or be- tween obstacles, within a factory, on a building site, or between a dock and a ship's hold. But developing the driving skills takes time and is expensive. Also even highly trained drivers can make mistakes. Furthermore drivers will typically err on the side of safety at the cost of longer manoeuvre times. Often drivers will also rely on at least one assistant (a person) to guide the load, especially for final positioning. One view of improving automatic control, therefore, is to try to understand what experienced crane drivers do intuitively, defining when to accelerate and to decelerate, and for planar trolleys, where and when to change direction and how quickly. But fully understanding what a human operator does is far from easy, and implementing this in a robust automatic control system is a further challenge.

This paper is about automating the process of moving the gantry of a crane to control the motion of a load when the load is dynamically complex. Specifically the paper is about a technique to solve this challenge based on the idea of mechanical waves. The technique is shown to have many advantages over existing approaches, including robustness to changes in the system dynamics, known or unknown; ease of implementation; and general applicability. 
The reference inputs are requested motions to the system, that is, desired controlled motions of the load. These inputs could be desired crane positions or velocities over time. (Specifying one, of course, implicitly specifies the other.) Here it is assumed that the reference inputs are position control requests for the load motion over time. Often the problem is one of getting the load to track a desired trajectory. Many papers measure performance by the tracking ability of their control strategies (Sun et al., 2013; Kim and Singhose, 2010; Manning et al., 2010; Neupert et al., 2010; Forest et al., 2001; Vaughan et al., 2011).

In published work to date, the load and cable dynamics are frequently modelled as a simple or (at most) a double pendulum system moving in a plane. Abdel-Rahman et al. (2003) in a crane review article report that "most control strategies for this class of crane assume a planar gantry crane, utilize planar, linear models, and assume that the crane path, external forces, and control effort are all planar". The simple pendulum model considerably simplifies the dynamics, giving a swing frequency which is independent of the load mass. The simplification is not always appropriate however. Some researchers have recently modelled the loads as 3-D pendula to which various control techniques have been applied. However they generally assume a single, lumped-mass, load (Al-Garni et al., 1995; Sun et al., 2013; August et al., 2010; Yang and Yang, 2006; Antić et al., 2012; Cheng-Yuan et al., 2006; Maghsoudi et al., 2012; Chen et al., 2005; Schulze and Chang, 2010; Zhong, 2011). The most dynamically advanced developments in crane controllers in 3-D assume point mass loads, or at most, a rod-like body hanging from the trolley.

Some papers report work on controlling double-pendulum cranes (Kim and Singhose, 2006; Kim and Singhose, 2010; Masoud and Nayfeh, 2003; Sawodny et al., 2002; Manning et al., 2010; Tanaka and Kouno, 1998; Cheng-jun et al., 2009; Singhose and Towel, 1998; Kenison and Singhose, 1999; Dan and $\mathrm{Li}, 2008$ ) but their simulations or experiments are planar rather than spatial. Manning et al. (2010), for example, present a dynamic model of bridge cranes with distributedmass loads as a planar double pendulum. This work is an example of the use of input shapers. In general, the input shaper design depends on knowing the natural frequencies of the flexible system to be controlled. See Kenison and Singhose (1999) for example.

The authors found no research which considered the control of a 3-D double-pendulum crane involving rigid body dynamics, so this aspect of the work is considered novel. Also novel is the application of wave ideas to this control problem, although it had previously been applied to a simple, onedegree of freedom gantry crane leading to a robust control performance (O'Connor, 2003). In this work it is shown that "wave-based control", or WBC, can be applied successfully to controlling more complex dynamical systems in 3-D, such as controlling the double-pendulum load, retaining many of the advantages that WBC demonstrated in controlling simpler systems.
The paper treats the load as a distributed mass, with translational and swing rotational inertia effects about two axes. Furthermore, this distributed load is assumed to be hanging from a hook of significant mass, about which the load is free to swing. The hook in turn is modeled as a lumped mass, so that its 3-D translational inertia effects can be taken into account. The trolley is taken to move in the horizontal plane, with motion controllers for two perpendicular axes. Three simplifying assumptions or restrictions are made. Firstly, the effects of cable hoisting are not considered. Secondly, the mass of the cable is assumed to be negligible. Thirdly, spin rotation of the load (as opposed to swinging in either planar direction) is neglected. (The authors are confident that WBC can easily be adapted to work without these assumptions, but the present paper assumes them to make the flexible dynamics more manageable.) The entire system has 6 degrees of freedom, four of which are determined by the system dynamics and two of which are controlled, input variables. Figure 1 shows a schematic of the system model and the variables used as coordinates in the dynamic model.

As a control technique, WBC has been successfully applied to various flexible mechanical systems. It sees the actuator motion (in this case the motion of the trolley) as launching a disturbance, or mechanical wave, into the flexible system, while responding to waves coming back from the system, usually trying to absorb them (O'Connor, 2003, 2007; O'Connor et al., 2009; O'Connor and Fumagalli, 2009; O'Connor and McKeown, 2008; McKeown, 2009). This launching and absorbing are considered to be happening simultaneously. These notional motion waves have DC components (or net displacement components), which, on passing thought the flexible system, from actuator to tip and back again, leave behind the desired net displacement, while simultaneously controlling vibrations. The control system decides on the launch wave net displacement, usually setting it to half the reference displacement. The returning motion wave from the system is measured and is added to the launch wave, and this combination forms the input to the trolley motion controllers.

The returning-wave component has two important effects. Firstly, it causes the trolley to absorb the vibration (that is, reduce the load swing) both during the manoeuvre and on arrival at the final position. Secondly, in the absence of significant external, disturbing forces, the net motion associated with the absorbing motion will exactly equal the net motion of the launch wave, so that it makes up the second half of the reference displacement. It will be shown that this fairly simple idea produces rapid, robust control, which is easy to implement, of low order, and which does not depend on having a precise system model, or ideal actuator response, or position sensing at the load. 


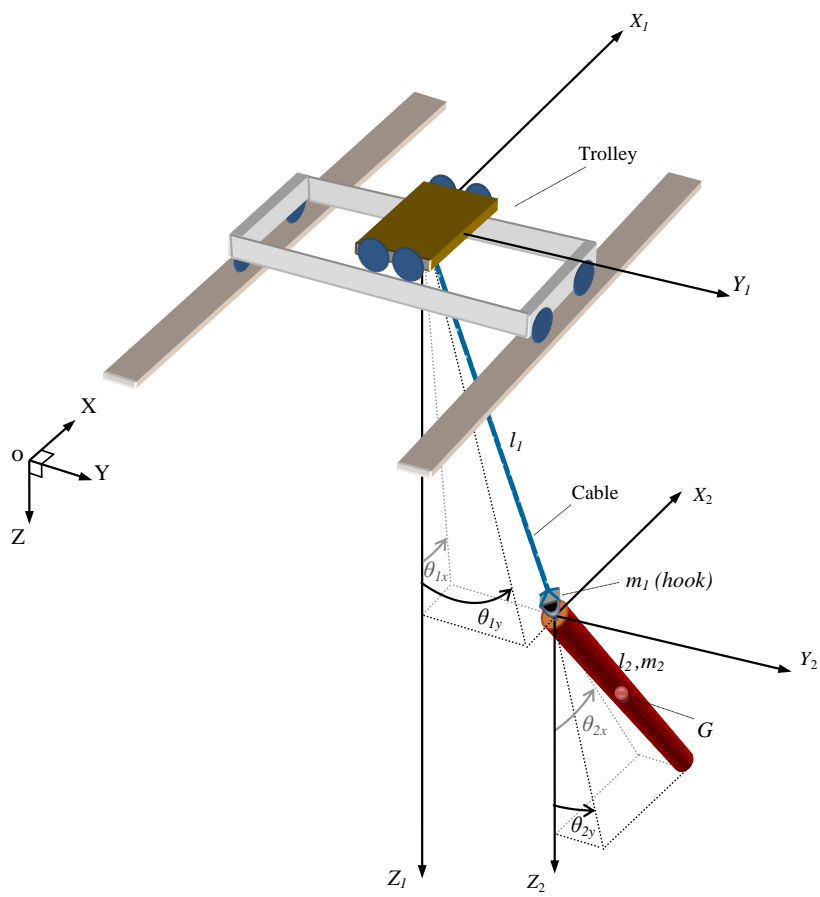

Figure 1. Representation of the 3-D double pendulum gantry crane.

\section{3-D model of system dynamics}

Figure 1 represents the entire physical system as modeled, including trolley, rails, cable, hook and hanging rigid body payload.

The trolley can move in both $x$ and $y$ directions simultaneously, so that it can follow an arbitrary path in the $X-Y$ plane in response to input signals to the trolley position subcontrollers. The reference system with coordinates $X_{1} Y_{1} Z_{1}$ is attached to the trolley, with $Z_{1}$ vertically downwards. The hook is connected to the trolley by a light, flexible cable, with the cable mass considered negligible in comparison with that of the hook and the load. The hook is modeled as a point mass $\left(m_{1}\right)$, the kinetic energy of which is therefore purely translational. Also since cable hoisting is ignored, the cable length is taken as a constant, $l_{1}$, so the exact position of the hook can be determined by the angle of the cable in space. This angle is measured by two variables, $\theta_{1 x}$ and $\theta_{1 y}$, which are the projections of the cable onto the $X-Z$ plane and $Y-Z$ planes, respectively. A second reference frame with coordinates system $\mathrm{X}_{2} Y_{2} Z_{2}$, parallel to the first, has its origin at the hook, and is used to describe the swing rotation of the load, again using two angles, $\theta_{2 x}$ and $\theta_{2 y}$, which are projections of the load angle onto the $X-Z$ and $Y-Z$ planes, respectively.

The instantaneous location of hook with respect to an inertial (space) coordinate system is given by

$\left\{\begin{array}{l}x_{H}=x_{T}+l_{1} \sin \theta_{1 x} \\ y_{H}=y_{T}+l_{1} \cos \theta_{1 x} \sin \theta_{1 y} \\ z_{H}=l_{1} \cos \theta_{1 x} \cos \theta_{1 y}\end{array}\right.$ where $x_{T}$ and $y_{T}$ are the trolley's position in the inertial reference frame. The position of the mass centre, $G$, of the swinging load is given by

$\left\{\begin{array}{l}x_{G}=x_{T}+l_{1} \sin \theta_{1 x}+\frac{l_{2}}{2} \sin \theta_{2 x} \\ y_{G}=y_{T}+l_{1} \cos \theta_{1 x} \sin \theta_{1 y}+\frac{l_{2}}{2} \cos \theta_{2 x} \sin \theta_{2 y} \\ z_{G}=l_{1} \cos \theta_{1 x} \cos \theta_{1 y}+\frac{l_{2}}{2} \cos \theta_{2 x} \cos \theta_{2 y}\end{array}\right.$

The equations of motion are obtained from Lagrange's equation

$\frac{\mathrm{d}}{\mathrm{d} t}\left(\frac{\partial L}{\partial \dot{q}_{i}}\right)-\left(\frac{\partial L}{\partial q_{i}}\right)=0$

The generalized coordinates, $q_{i}$, are here taken as the four independent variables $\theta_{1 x}, \theta_{1 y}, \theta_{2 x}$ and $\theta_{2 y}$. The trolley position variables $x_{T}$ and $y_{T}$ are considered as input variables, used to control the attached flexible system, so four equations of motion are required. If $m_{1}$ is the mass of the hook and $m_{2}$ the mass of the load, total potential energy is

$$
\begin{aligned}
U & =m_{1} g l_{1}\left(1-\cos \theta_{1 x} \cos \theta_{1 y}\right)+m_{2} g \\
& {\left[l_{1}\left(1-\cos \theta_{1 x} \cos \theta_{1 y}\right)+\frac{l_{2}}{2}\left(1-\cos \theta_{2 x} \cos \theta_{2 y}\right)\right] }
\end{aligned}
$$

where $g$ is the acceleration due to gravity. The total kinetic energy may describe as

$T=\frac{1}{2} m_{1} \vec{v}_{H} \vec{v}_{H}+\frac{1}{2} m_{2} \vec{v}_{G} \vec{v}_{G}+\frac{1}{2} \tilde{\omega}\left[I_{G}\right] \tilde{\omega}$

where $\vec{v}_{H}$ is the hook velocity, $\vec{v}_{G}$ is the velocity of point $G$, and $\tilde{\omega}$ is the total angular velocity of the load. The linear velocities are the derivatives of Eqs. (1) and (2), which may be expressed in the form

$\vec{v}_{H}:\left\{\begin{array}{l}\dot{x}_{H}=\dot{x}_{T}+l_{1} \dot{\theta}_{1 x} \cos \theta_{1 x} \\ \dot{y}_{H}=\dot{y}_{T}-l_{1} \dot{\theta}_{1 x} \sin \theta_{1 x} \sin \theta_{1 y}+l_{1} \dot{\theta}_{1 y} \cos \theta_{1 x} \cos \theta_{1 y} \\ \dot{z}_{H}=-l_{1} \dot{\theta}_{1 x} \sin \theta_{1 x} \cos \theta_{1 y}-l_{1} \dot{\theta}_{1 y} \cos \theta_{1 x} \sin \theta_{1 y}\end{array}\right.$

$\vec{v}_{G}:\left\{\begin{array}{l}\dot{x}_{G}=\dot{x}_{H}+\frac{l_{2}}{2} \dot{\theta}_{2 x} \cos \theta_{2 x} \\ \dot{y}_{G}=\dot{y}_{H}-\frac{l_{2}}{2} \dot{\theta}_{2 x} \sin \theta_{2 x} \sin \theta_{2 y}+\frac{l_{2}}{2} \dot{\theta}_{2 y} \cos \theta_{2 x} \cos \theta_{2 y} \\ \dot{z}_{G}=\dot{z}_{H}-\frac{l_{2}}{2} \dot{\theta}_{2 x} \sin \theta_{2 x} \cos \theta_{2 y}-\frac{l_{2}}{2} \dot{\theta}_{2 y} \cos \theta_{2 x} \sin \theta_{2 y}\end{array}\right.$

To quantify the rotational kinetic energy of the load requires its angular velocity $\tilde{\omega}$ and moment of inertia tensor, $\left[I_{G}\right]$, to be expressed in a common coordinate system. The load inertia tensor is most conveniently expressed using a coordinate system $X_{2 c} Y_{2 c} Z_{2 c}$ fixed to the load and along its principal axes, so that

$\left[I_{G}\right]=\left[\begin{array}{lll}I_{x x} & 0 & 0 \\ 0 & I_{y y} & 0 \\ 0 & 0 & I_{z z}\end{array}\right]_{X_{2 c} Y_{2 c} Z_{2 c}}$

If so, $\tilde{\omega}$ should also be expressed in the same coordinate system. As shown in Fig. 2, the coordinate system $X_{2 a} Y_{2 a} Z_{2 a}$ 

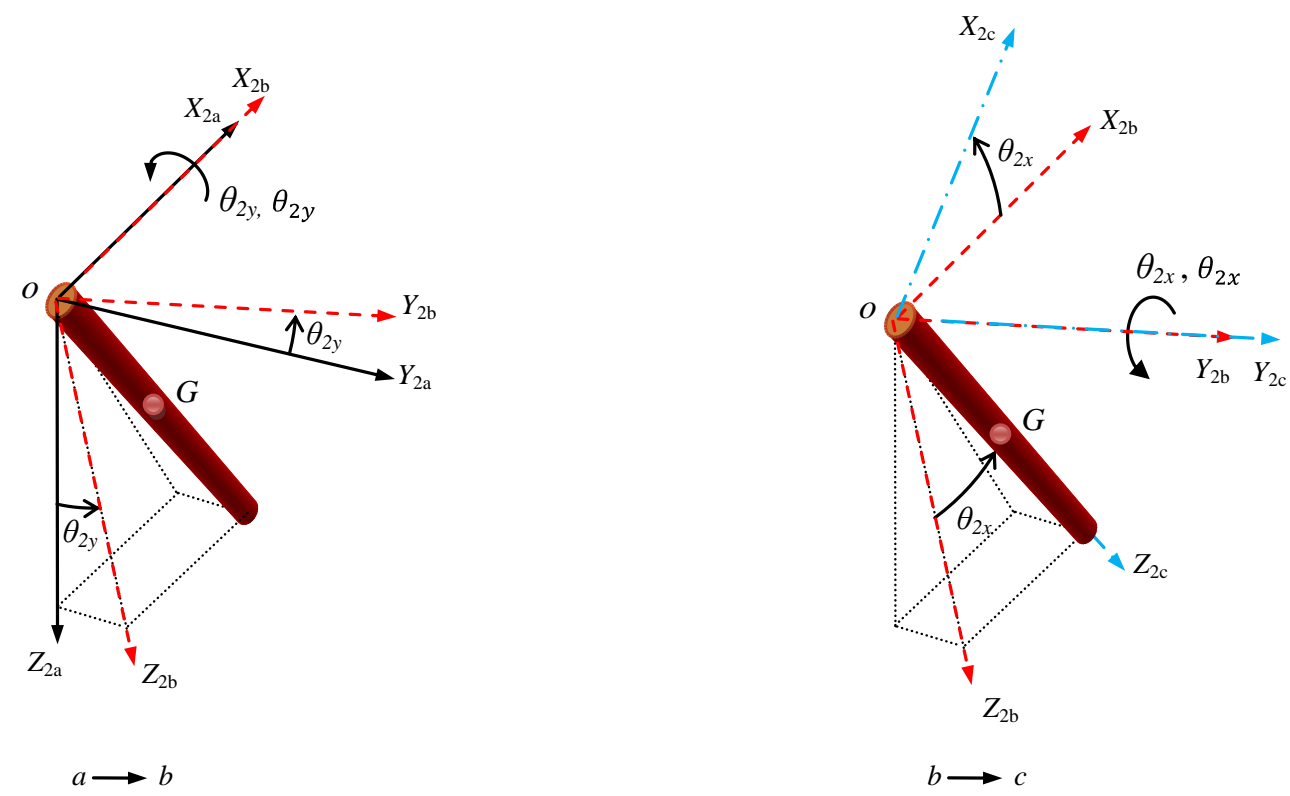

Figure 2. Representation of successive rotations to reach the body coordinates of the hanging load.

moves through two rotations about the main axes to become aligned to the load body axis $X_{2 c} Y_{2 c} Z_{2 c}$. The angular velocity $(\tilde{\omega})$ according to Fig. 2 is defined as

$\tilde{\omega}=-\dot{\theta}_{2 y} \hat{i}_{2 b}+\dot{\theta}_{2 x} \hat{j}_{2 c}$

The transformation from $X_{2 b} Y_{2 b} Z_{2 b}$ to $X_{2 c} Y_{2 c} Z_{2 c}$ or $T_{2 b \rightarrow 2 c}$ is

$T_{(2 b \rightarrow 2 c)}=\left[\begin{array}{ccc}\cos \theta_{2 x} & 0 & -\sin \theta_{2 x} \\ 0 & 1 & 0 \\ \sin \theta_{2 x} & 0 & \cos \theta_{2 x}\end{array}\right]$

So finally $\tilde{\omega}$ can be expressed in $X_{2 c} Y_{2 c} Z_{2 c}$ as

$\tilde{\omega}=\left[\begin{array}{c}-\dot{\theta}_{2 y} \cos \theta_{2 x} \\ \dot{\theta}_{2 x} \\ -\dot{\theta}_{2 y} \sin \theta_{2 x}\end{array}\right]_{X_{2 c} Y_{2 c} Z_{2 c}}$

The rotational kinetic energy of the hanging load, $T_{\omega}$, is then expressible as

$$
\begin{aligned}
T_{\omega} & =\frac{1}{2} \tilde{\omega}\left[I_{G}\right] \tilde{\omega}=\frac{1}{2} I_{x x}\left(\dot{\theta}_{2 y}\right)^{2}\left(\cos \theta_{2 x}\right)^{2}+\frac{1}{2} I_{y y}\left(\dot{\theta}_{2 x}\right)^{2} \\
& +\frac{1}{2} I_{z z}\left(\dot{\theta}_{2 y}\right)^{2}\left(\sin \theta_{2 x}\right)^{2}
\end{aligned}
$$

Substituting Eqs. (6), (7) and (12) into Eq. (5) gives the total kinetic energy of system. Then this $T$ from Eq. (5) minus $U$ from Eq. (4) gives the Lagrangian, $L=T-U$ to be used in Eq. (3), with $q_{i}$ equal, in turn, to each of the four angles $\theta_{1 x}$, $\theta_{1 y}, \theta_{2 x}$ and $\theta_{2 y}$, giving four equations of motion, which after simplification become

$\left(m_{1} l_{1}^{2}+m_{2} l_{1}^{2}\right) \ddot{\theta}_{1 x}+\left(m_{1}+m_{2}\right) l_{1} \ddot{x}_{T} \cos \theta_{1 x}-\left(m_{1}+m_{2}\right) l_{1} \ddot{y}_{T}$ $\sin \theta_{1 x} \sin \theta_{1 y}+\left(m_{1}+m_{2}\right) l_{1}^{2}\left(\dot{\theta}_{1 y}\right)^{2} \cos \theta_{1 x} \sin \theta_{1 x}+\frac{1}{2} m_{2} l_{1} l_{2} \ddot{\theta}_{2 x}$ $\cos \theta_{1 x} \cos \theta_{2 x}-\frac{1}{2} m_{2} l_{1} l_{2}\left(\dot{\theta}_{2 x}\right)^{2} \cos \theta_{1 x} \sin \theta_{2 x}+\frac{1}{2} m_{2} l_{1} l_{2} \ddot{\theta}_{2 x}$ $\sin \theta_{1 x} \sin \theta_{2 x} \cos \left(\theta_{1 y}-\theta_{2 y}\right)+\frac{1}{2} m_{2} l_{1} l_{2}\left(\dot{\theta}_{2 x}\right)^{2}+\left(\dot{\theta}_{2 y}\right)^{2} \sin \theta_{1 x}$ $\cos \theta_{2 x} \cos \left(\theta_{2 y}-\theta_{1 y}\right)+\frac{1}{2} m_{2} l_{1} l_{2} \ddot{\theta}_{2 y} \sin \theta_{1 x} \cos \theta_{2 x} \sin \left(\theta_{2 y}-\theta_{1 y}\right)$ $+m_{2} l_{1} l_{2} \dot{\theta}_{2 x} \dot{\theta}_{2 y} \sin \theta_{1 x} \sin \theta_{2 x} \sin \left(\theta_{1 y}-\theta_{2 y}\right)+\left(m_{1}+m_{2}\right) g l_{1}$ $\sin \theta_{1 x} \cos \theta_{1 y}=0$

$\left(m_{1}+m_{2}\right) l_{1}^{2}\left(\cos \theta_{1 x}\right)^{2} \ddot{\theta}_{1 y}-2\left(m_{1}+m_{2}\right) l_{1}^{2} \dot{\theta}_{1 x} \dot{\theta}_{1 y} \sin \theta_{1 x} \cos \theta_{1 x}$ $+\left(m_{1}+m_{2}\right) l_{1} \ddot{y}_{T} \cos \theta_{1 x} \cos \theta_{1 y}+\frac{1}{2} m_{2} l_{1} l_{2} \ddot{2}_{2 y} \cos \theta_{1 x} \cos \theta_{2 x}$ $\cos \left(\theta_{1 y}-\theta_{2 y}\right)+\frac{1}{2} m_{2} l_{1} l_{2}\left(\dot{\theta}_{2 x}\right)^{2}+\left(\dot{\theta}_{2 y}\right)^{2} \cos \theta_{1 x} \cos \theta_{2 x}$ $\sin \left(\theta_{1 y}-\theta_{2 y}\right)+\frac{1}{2} m_{2} l_{1} l_{2} \ddot{\theta}_{2 x} \cos \theta_{1 x} \sin \theta_{2 x} \sin \left(\theta_{1 y}-\theta_{2 y}\right)$ $-m_{2} l_{1} l_{2} \dot{\theta}_{2 x} \dot{\theta}_{2 y} \cos \theta_{1 x} \sin \theta_{2 x} \cos \left(\theta_{1 y}-\theta_{2 y}\right)+\left(m_{1}+m_{2}\right)$ $g l_{1} \sin \theta_{1 y} \cos \theta_{1 x}=0$ 


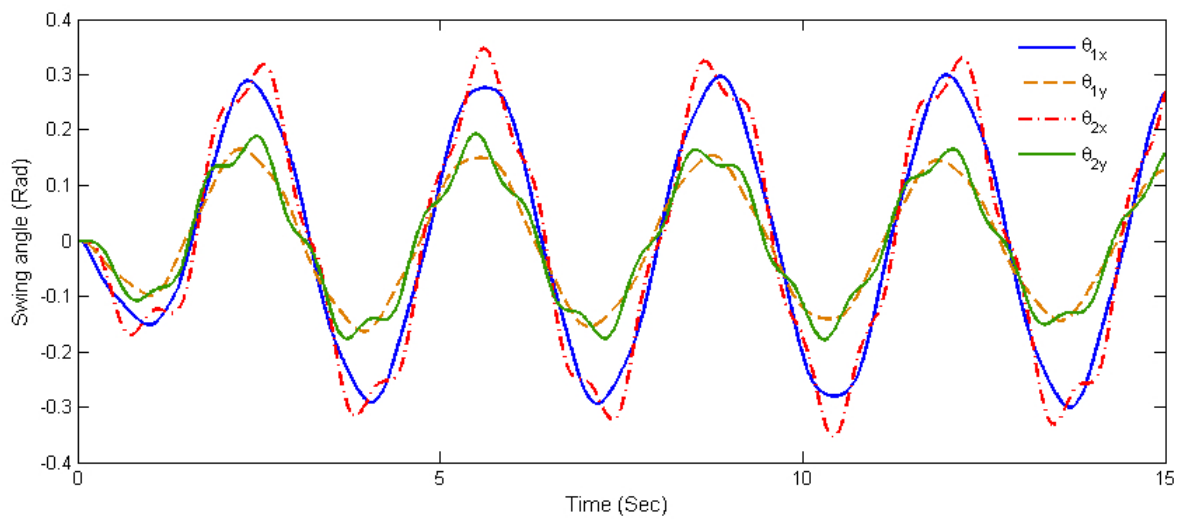

Figure 3. Dynamic response of 3-D double pendulum gantry crane with 4 degree of freedom.

$\left(m_{2} \frac{l_{2}^{2}}{4}+I_{y y}\right) \ddot{\theta}_{2 x}+\frac{1}{2} m_{2} l_{2} \ddot{x}_{T} \cos \theta_{2 x}-\frac{1}{2} m_{2} l_{2} \ddot{y}_{T} \sin \theta_{2 x}$

$\sin \theta_{2 y}+\frac{1}{2} m_{2} l_{1} l_{2} \ddot{\theta}_{1 x} \cos \theta_{1 x} \cos \theta_{2 x}-\frac{1}{2} m_{2} l_{1} l_{2}\left(\dot{\theta}_{1 x}\right)^{2}$

$\sin \theta_{1 x} \cos \theta_{2 x}+\frac{1}{2} m_{2} l_{1} l_{2} \ddot{\theta}_{1 x} \sin \theta_{1 x} \sin \theta_{2 x} \cos \left(\theta_{1 y}-\theta_{2 y}\right)$

$+\frac{1}{2} m_{2} l_{1} l_{2}\left(\dot{\theta}_{1 x}\right)^{2}+\left(\dot{\theta}_{1 y}\right)^{2} \cos \theta_{1 x} \sin \theta_{2 x} \cos \left(\theta_{1 y}-\theta_{2 y}\right)+\frac{1}{2}$

$m_{2} l_{1} l_{2} \ddot{\theta}_{1 y} \cos \theta_{1 x} \sin \theta_{2 x} \sin \left(\theta_{1 y}-\theta_{2 y}\right)-m_{2} l_{1} l_{2} \dot{\theta}_{1 y} \dot{\theta}_{1 x} \sin \theta_{1 x}$

$\sin \theta_{2 x} \sin \left(\theta_{1 y}-\theta_{2 y}\right)+m_{2} \frac{l_{2}^{2}}{4}\left(\dot{\theta}_{2 y}\right)^{2} \cos \theta_{2 x} \sin \theta_{2 x}+\left(I_{x x}-I_{z z}\right)$

$\left(\dot{\theta}_{2 y}\right)^{2} \cos \theta_{2 x} \sin \theta_{2 x}+m_{2} g \frac{l_{2}}{2} \sin \theta_{2 x} \cos \theta_{2 y}=0$

$\left(m_{2} \frac{l_{2}^{2}}{4}\left(\cos \theta_{2 x}\right)^{2}+I_{x x}\left(\cos \theta_{2 x}\right)^{2}+I_{z z}\left(\sin \theta_{2 x}\right)^{2}\right) \ddot{\theta}_{2 y}-m_{2} \frac{l_{2}^{2}}{2} \dot{\theta}_{2 x} \dot{\theta}_{2 y}$ $\cos \theta_{2 x} \sin \theta_{2 x}+\frac{1}{2} m_{2} l_{2} \ddot{y}_{T} \cos \theta_{2 x} \cos \theta_{2 y}+\frac{1}{2} m_{2} l_{1} l_{2} \ddot{\theta}_{1 x} \sin \theta_{1 x}$ $\cos \theta_{2 x} \sin \left(\theta_{2 y}-\theta_{1 y}\right)+\frac{1}{2} m_{2} l_{1} l_{2}\left\{\left(\dot{\theta}_{1 x}\right)^{2}+\left(\dot{\theta}_{1 y}\right)^{2}\right\} \cos \theta_{1 x} \cos$

$\theta_{2 x} \sin \left(\theta_{2 y}-\theta_{1 y}\right)+\frac{1}{2} m_{2} l_{1} l_{2} \ddot{\theta}_{1 y} \cos \theta_{1 x} \cos \theta_{2 x} \cos \left(\theta_{1 y}-\theta_{2 y}\right)$

$-m_{2} l_{1} l_{2} \dot{\theta}_{1 x} \dot{\theta}_{1 y} \sin \theta_{1 x} \cos \theta_{2 x} \cos \left(\theta_{2 y}-\theta_{1 y}\right)+\left(I_{z z}-I_{x x}\right)$

$\dot{\theta}_{2 x} \dot{\theta}_{2 y} \sin \left(2 \theta_{2 x}\right)+m_{2} \frac{l_{2}}{2} g \cos \theta_{2 x} \sin \theta_{2 y}=0$

These four, highly coupled, equations of motion capture the full system dynamics. In the modeling, no small-angle approximations were made to keep the model accurate even for large swing angles. These four equations can be integrated numerically from given initial conditions to describe the time evolution of the system. The trolley motion components are considered as inputs, defining $\ddot{x}_{T}$ and $\ddot{y}_{T}$ in these equations. As an example, Fig. 3 shows the behavior of the system for an arbitrary planar movement of the trolley with no damping
Table 1. Values chosen for the 3-D double pendulum system.

\begin{tabular}{llll}
\hline$m_{1}(\mathrm{~kg})$ & $m_{2}(\mathrm{~kg})$ & $l_{1}(\mathrm{~m})$ & $l_{2}(\mathrm{~m})$ \\
\hline 1 & 50 & 1 & 2 \\
\hline
\end{tabular}

or control action. The chosen system parameters (which can be arbitrarily chosen) are given in Table 1 .

While the input motion of the trolley takes no longer than two seconds, the system keeps swinging indefinitely, with multiple frequency components. Alternatively, rather than by trolley motion, the system can be set in motion by giving it initial angular displacements and/or velocities, with the trolley stationary. If desired this can be done in such a way that the subsequent motion corresponds to the mode shapes, at each of four natural frequencies.

With the crane model behaving as expected, the WBC ideas are now developed and used to control the swinging load by controlling the trolley motion.

\section{Wave-based approach to crane control}

From a control perspective, the trolley is a single actuator attempting to control a flexible system of relatively complex dynamics. The system is under-actuated, with more degrees of freedom than actuators. The actuator does not act directly on the load position and orientation, but must work through the intervening flexible dynamics, of cable and hook, to try to achieve a target motion of the load. The actuator motion is in two perpendicular directions, the load can swing in 3-D, and the motion components are strongly coupled.

The control method adopted here, wave-based control, is a generic approach, which does not depend on having an accurate system model. It uses feedback, but the feedback measurements are taken not at the system output (here, the load position and orientation) but at the actuator (the trolley, in this case). Thus the actuator and sensing are collocated, with 


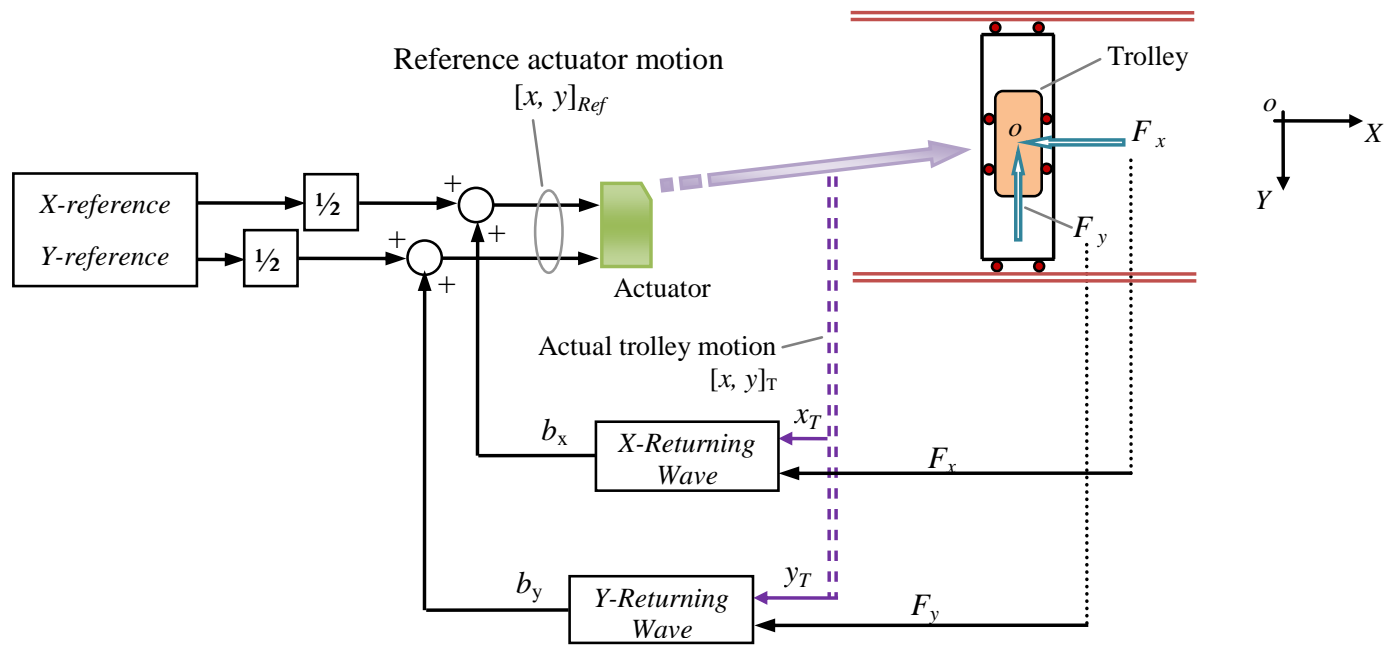

Figure 4. WBC plan for gantry crane with planar-moving trolley.

the consequent stability advantages. To date WBC has been used successfully to control 1-D rectilinear lumped flexible systems, 2-D flexible mass-spring arrays either beam-like or arranged in a grid, laterally flexing manipulators, and a simple pendulum gantry crane as already mentioned (O'Connor, 2003).

In the current case, there are two inputs actuating the trolley motion in two orthogonal directions. So the trolley should be given two reference displacements, in the $x$ and $y$ directions, and two corresponding returning waves, $b_{x}$ and $b_{y}$, to achieve wave absorption in the two directions under WBC. Figure 4 illustrates a version of a general scheme for positioning the trolley, along with the payload, to a target position in the plane.

Here the returning displacement waves are defined and measured as

$b_{x}=\frac{1}{2}\left[x_{T}-\frac{1}{Z_{x}} \int_{0}^{t} F_{x} \mathrm{~d} t\right]$

$b_{y}=\frac{1}{2}\left[y_{T}-\frac{1}{Z_{y}} \int_{0}^{t} F_{y} \mathrm{~d} t\right]$

where $x_{T}$ and $y_{T}$ are the trolley position coordinates, $F_{x}$ and $F_{y}$ are the horizontal components in the $x$ and $y$ directions of the cable force measured at the trolley, and $Z_{x}, Z_{y}$ are impedance terms. References O'Connor (2003) and McKeown (2009) outline how such expressions for the returning waves can be developed. Here we simply note the following two features.

First, for rest-to-rest motion, from time $t=0$ to some final time $t$, as the initial and final momenta are zero, the force integrals must be zero. So the final values of $b_{x}$ and $b_{y}$ will be half the trolley displacements, or $1 / 2 x_{T}$ and $1 / 2 y_{T}$. Note that this result holds regardless of the values of the impedances $Z_{x}$ and $Z_{y}$. The second observation is that while $F_{x}$ and $F_{y}$ are changing, the effect of adding $b_{x}$ and $b_{y}$ to the trolley's motion is to make the trolley act as a viscous damper with damping coefficient $Z_{x}, Z_{y}$ in response to the cable forces.

The values of impedance are not critical to the control scheme. In this work both impedances were set to

$Z=\left(m_{1}+m_{2}\right) \sqrt{\frac{g}{\left(l_{1}+l_{2}\right)}}$

Variations in the values of $Z$ cause small variations in the transient part of the responses. So $Z$ can be used as a parameter with which to fine-tune the transient, for example to improve a specific performance measure (e.g. rise time, overshoot, or settling time), as appropriate for a given application, invariably at the cost of a slight degradation of some other transient performance measure (although always retaining the zero steady-state error).

The force components $F_{x}$ and $F_{y}$ should ideally correspond to the horizontal components of the cable tension, including the dynamic effects of the acceleration of the load mass. For most purposes, however, they can be approximated by assuming that the cable tension is equal to the load weight, and then $F_{x}$ and $F_{y}$ from Fig. 1 can be taken as

$F_{x}=\frac{-\left(m_{1}+m_{2}\right) g \tan \theta_{1 x}}{\cos \theta_{1 y}}$

$F_{y}=-\left(m_{1}+m_{2}\right) g \tan \theta_{1 y}$

These approximations were used in obtaining the results below. 


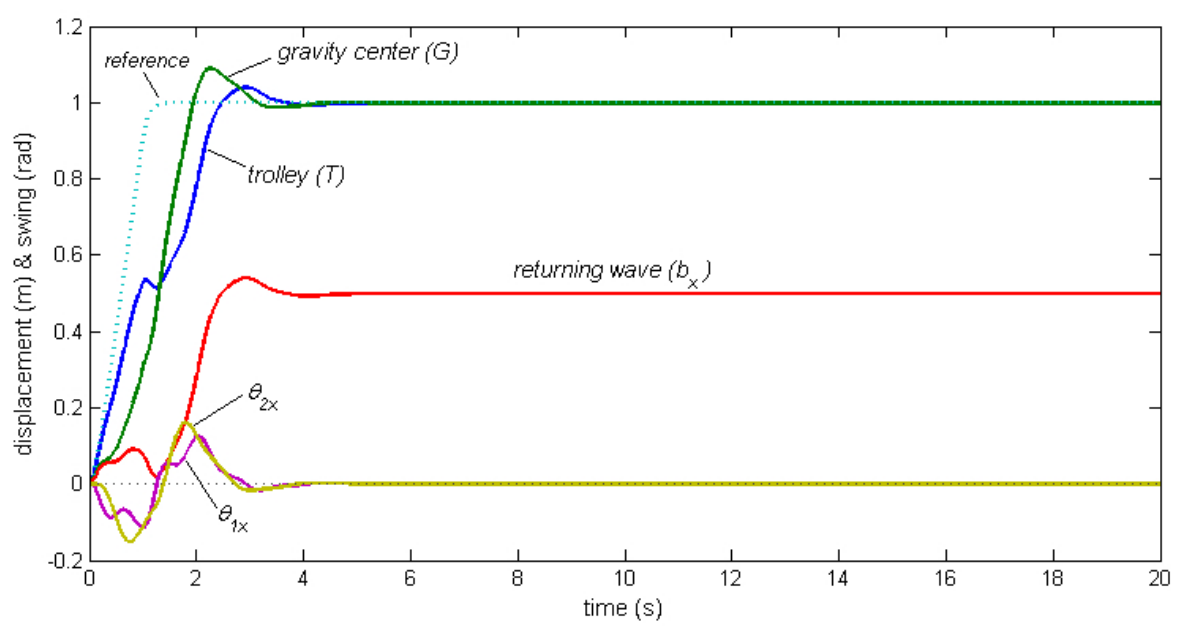

Figure 5. Gantry crane response to a $1 \mathrm{~m}$ displacement in $x$ direction using WBC.

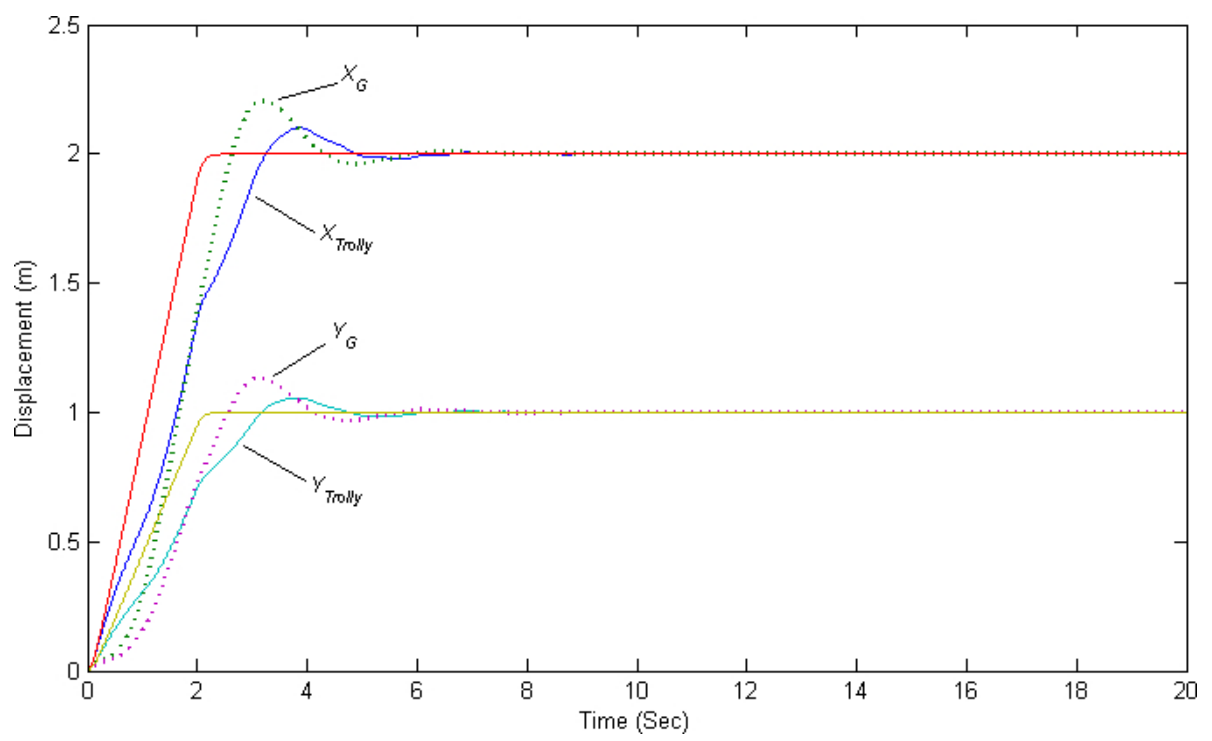

Figure 6. Response to planar trolley motion under WBC (sub $G$ : mass centre of distributed load).

\section{Results}

In the first manoeuvre the reference is a simple ramp (or constant velocity) displacement of one meter in the $x$ direction, with no motion in the $y$ direction. Figure 5 depicts the response of both the trolley and the centre of mass of the payload, $G$, under the control system of Fig. 4. Also the swinging angles of the cable (in both directions) and of the load due to this excitation are displayed in radians. The trolley can be seen to settle quickly at the target displacement with an initial overshoot of less than $10 \%$. The centre of mass of the hanging load, at $2 \mathrm{~m}$ from the trolley, comes to rest rapidly, with little swing as shown by $\theta_{1 x}$ and $\theta_{2 x}$, and with an overshoot of about $10 \%$. Clearly the suspension swinging dies out soon after the trolley reaches the target. Also shown is the returning wave, $b_{x}$, which provides the swing absorption and settles at half the target displacement. (In this case there is no $b_{y}$ as all the motion is in the $x$ direction.)

In the next example, the reference input is a simultaneous combination of a ramp up to $2 \mathrm{~m}$ in the $x$ direction and a ramp of a different slope up to one meter in the $y$ direction. Figure 6 shows the response. The trolley comes to rest gently with no steady-state error while quickly absorbing all oscillations. Figure 7 shows how the swing angles of the double pendulum are actively damped. As the model has no damping built in, the damping is being achieved entirely by the trolley motion which is simultaneously moving the system to the target displacement as seen in Fig. 6. The periodic time of the lowest mode of vibration is of the order of $4 \mathrm{~s}$. After $10 \mathrm{~s}$, that is within two and a half times the periodic time after arrival at the target, all oscillations have been reduced from the 


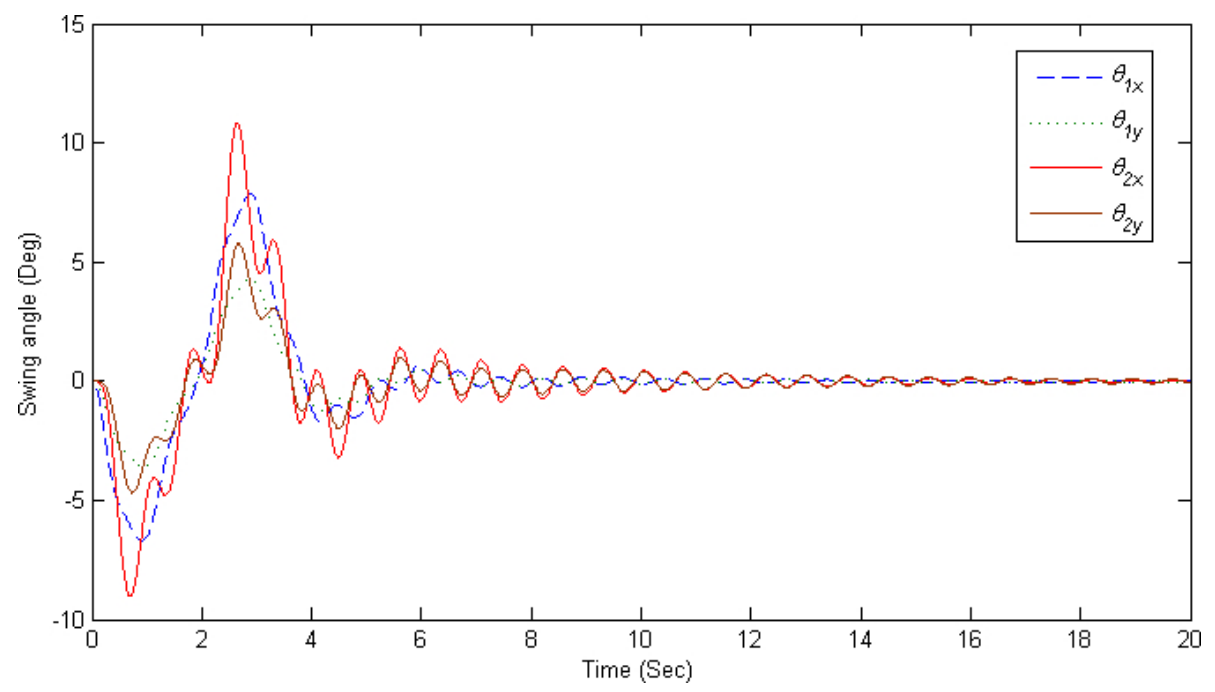

Figure 7. Swing angles for manoeuver of Fig. 6, showing active damping.

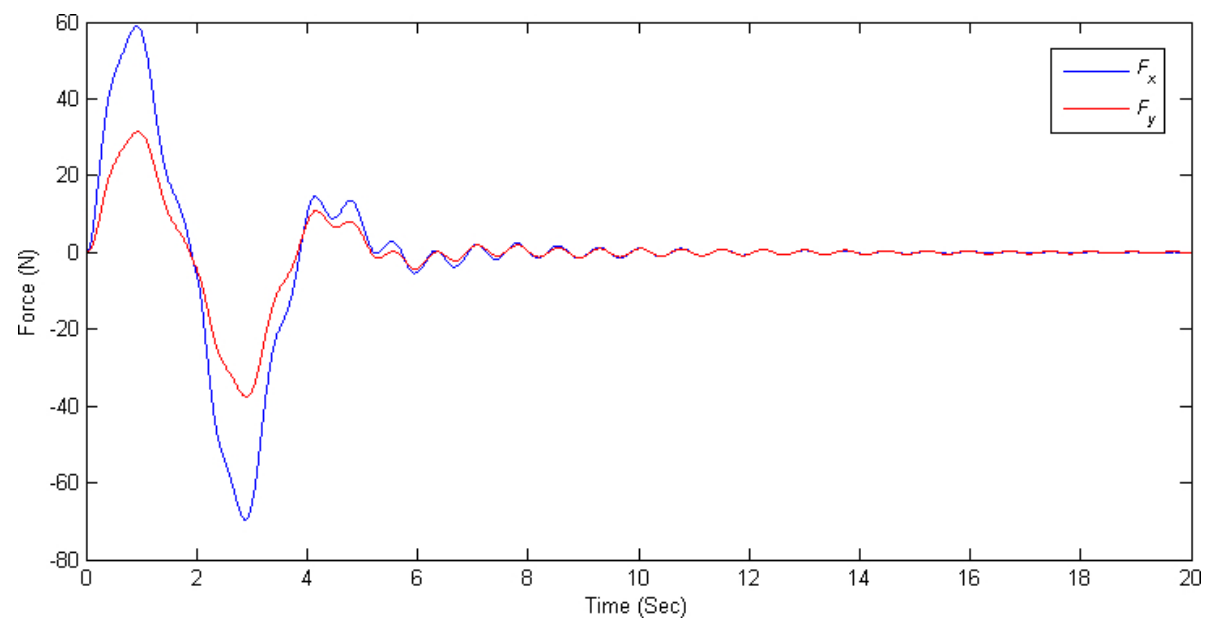

Figure 8. Reduction of forces acting on trolley through WBC process.

maximum value of about 10 degrees to less than 2 degrees and they are decaying steadily.

Figure 8 shows the horizontal force components between trolley and cable for the same manoeuvre. The main accelerations and decelerations occur within $4 \mathrm{~s}$, and after about $6 \mathrm{~s}$ the force amplitudes diminish to less than $3 \%$ of the initial peaks. Again WBC is using the forces to combine position control and active vibration damping very effectively.

Figure 9 shows the effect of choosing different values of the impedance parameter $Z$, as the only control parameter to be tuned, for the single-input manoeuvre of Fig. 5. The reference impedance $Z=Z_{\text {eq }}$ is as in Eq. (19), which is the value used to obtain the results presented above. Despite a 12 -fold range in impedance values, the responses are good for all cases, showing a stable response, rapid transit and zero steady-state error in the final position. The best choice of $Z$ will depend on the priorities in the desired response.
For example, perhaps a good compromise between minimum overshoot and shortest settling time is when $Z_{x}=(0.75) Z_{\mathrm{eq}}$. For $Z_{x} \geq 2 Z_{\text {eq }}$ the various responses become almost indistinguishable (except around the half-way point). More could have been added for other values of $Z_{x}$, but they would have fallen on top of the curves shown. On the other hand for low values of impedance, say $Z_{x}<(0.5) Z_{\text {eq }}$, the trolley has a slow transient and slow convergence to the target position.

The robustness of the control response to variations in $Z$ also indirectly illustrates the robustness of WBC to changes in the system under control, whether these are known or unknown, whether modeled or not. For example, if incorrect values of masses or lengths are assumed in using Eq. (19), or these parameter change during operations, the control system still copes well.

In producing these results, the trolley was assumed to have ideal dynamics, that is, that it reproduces exactly and 


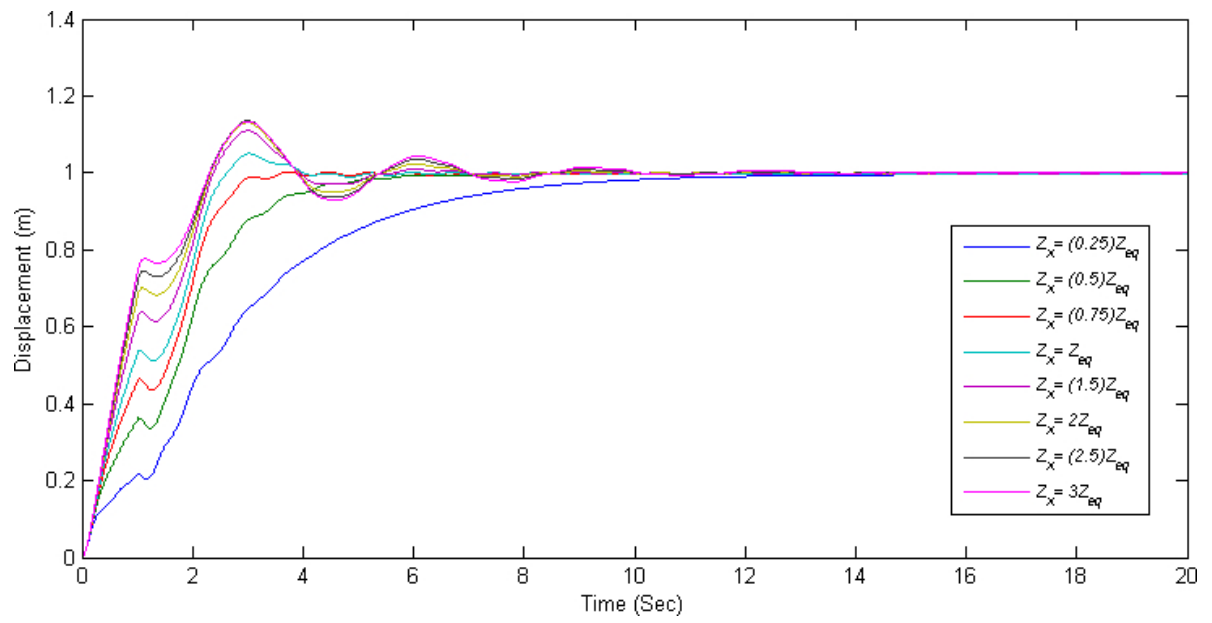

Figure 9. Trolley response to $1 \mathrm{~m} x$ direction input with different $Z$ values in the WBC.

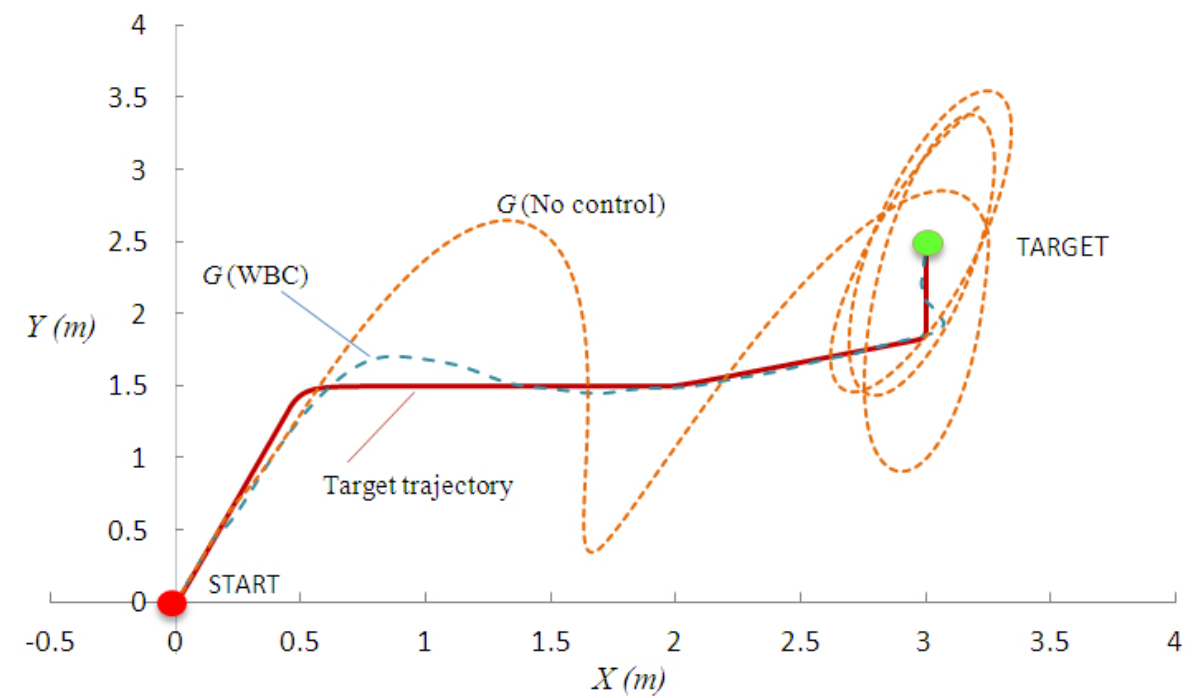

Figure 10. Trajectory tracking, plan view, showing the paths of the mass centre of the load.

immediately the motion requested by the WBC system of Fig. 4. However the system has also been tested with realistic trolley dynamics, where the trolley response shows some dynamic delay in achieving the requested motion. The control system still works well, with comparable results to those presented, provided that the steady state trolley error is zero. This robustness to the trolley's dynamic performance can be explained in part because the measurements used in the WBC control system, including Eqs. $(17,18)$ come after the trolley (see Fig. 4), using the values of position, $x_{T}, y_{T}$, and forces, $F_{x}, F_{y}$, actually achieved and experienced by the trolley.

Finally, in addition to the point-to-point manoeuvres above, input tracking and obstacle avoidance are considered. Figure 10 shows a plan view of a desired input trajectory, and the resulting path of the mass centre of the load, $G$, both un- der WBC and with no control. As can be seen, under WBC the tracking is very satisfactory.

\section{Conclusions}

The double-pendulum, distributed mass, gantry crane model assumed in this work has non-trivial dynamics, and represents a considerable advance on the simple pendulum model often used. A model was developed to capture these dynamics in three dimensions. The 2-D trolley motion has limited control authority over the 3-D suspended system. This paper explores how well a simple version of WBC can work to achieve load position control while damping the swinging in 3-D. The control strategy does not require details of the system model, and all the required measurements are taken at the trolley. The control law has one tuning parameter, a 
mechanical impedance term, whose value is not critical, but can be used to fine-tune the transient response as desired.

The results illustrate the power, simplicity, effectiveness and robustness of the control approach. For point to point manoeuvres in the plane, WBC proves very effective. It also performs well in trajectory tracking, often required for obstacle avoidance.

Future work will extend the approach to cranes with cable hoisting, to tower cranes in which the trolley moves on a rotating arm, and to jib or luffing cranes in which the arm rotates in the vertical plane. Initial results suggest that the same WBC strategy can be extended successfully to all such cases.

Edited by: A. Müller

Reviewed by: two anonymous referees

\section{References}

Abdel-Rahman, E. M., Nayfeh, A. H., and Masoud, Z. N.: Dynamics and Control of Cranes: A Review, J. Vib. Control, 9, 863-908, 2003.

Al-Garni, A. Z., Moustafa, K. A. F, and Javeed Nizami, S. S. A. K.: Optimal control of overhead cranes, Control Eng. Pract., 3, 1277-1284, 1995.

Antić, D., Jovanović, Z., Perić, S., Nikolić, S., Milojković, M., and Milošević, M.: Anti-Swing Fuzzy Controller Applied in a 3D Crane System, Engineering, Technology \& Applied Science Research (ETASR), 2, 196-200, 2012.

August, W., Ren, J., Notheis, S., Haase, T., Hein, B., and Wörn, H.: 3D Pendulum Swinging Control by an Industrial Robot Manipulator, 1-7, in: Proceeding of: ISR/ROBOTIK 2010, Proceedings for the joint conference of ISR 2010 (41st Internationel Symposium on Robotics) und ROBOTIK 2010 (6th German Conference on Robotics), 7-9 June 2010, Munich, Germany, 2010.

Chen, H., Gao, B., and Zhang, X.: Dynamical Modelling and Nonlinear Control of a 3D Crane, International Conference on Control and Automation (ICCA2005), Budapest, Hungary, 10851090, June 2005

Cheng-jun, D., Ping, D., Ming-lu, Z., and Yan-fang, Z.: Double Inverted Pendulum System Control Strategy Based On Fuzzy Genetic Algorithm, in: Proceedings of the IEEE International Conference on Automation and Logistics Shenyang, China, 13181323, August 2009.

Cheng-Yuan, C., Kou-Cheng, H., Kuo-Hung, C., and Guo-En, H.: An Enhanced Adaptive Sliding Mode Fuzzy Control for Positioning and Anti-Swing Control of the Overhead Crane System, IEEE International Conference on Systems, Man, and Cybernetics, Taipei, Taiwan, 992-997, October 2006.

Dan, Y. and Li, Z.: The Structure of HSIC System and Its Application on Arbitrary Switch Control of Double Pendulum, in: Proceedings of the 7th World Congress on Intelligent Control and Automation, Chongqing, China, 2810-2815, June 2008.

Forest, C., Frakes, D., and Singhose, W.: Input-Shaped Control of Gantry Cranes: Simulation and Curriculum Development, The 18th ASME DETC Biennial Conference on Mech. Vib. and Noise, 2001.

Kenison, M. and Singhose, W.: Input Shaper Design for DoublePendulum Planar Gantry Cranes, in: Proceedings of the I999
EEE International Conference on Control Applications, Kohala Coast-Island of Hawai'i, Hawai' i, USA, 539-544, August 1999.

Kim, D. and Singhose, W.: Reduction of Double-Pendulum Bridge Crane Oscillations, The 8th International Conference On Motion And Vibration Control (MOVIC 2006), Atlanta, GA, USA, 300305, 2006.

Kim, D. and Singhose, W.: Performance Studies Of Human Operators Driving Double-Pendulum Bridge Cranes, Control Eng. Pract., 18, 567-576, 2010.

Maghsoudi, M. J., Mohammed, Z., Pratiwi, A. F., Ahmad, N., and Husain, A. R.: An Experiment for Position and Sway Control of a 3D Gantry Crane, The 4th International Conference on Intelligent and Advanced Systems (ICIAS2012), 497-502, 2012.

Manning, R., Clement, J., Kim, D., and Singhose, W.: Dynamics and Control of Bridge Cranes Transporting DistributedMass Payloads, J. Dyn. Syst.-T. ASME, 132, 014505, doi:10.1115/1.4000657, 2010.

Masoud, Z. N. and Nayfeh, A. H.: Sway Reduction on Container Cranes Using Delayed Feedback Controller, Nonlinear Dynam., 34, 347-358, 2003.

McKeown, D. J.: Wave based Control of Elastic Mechanical Systems, Ph.D. Thesis, Department of Mechanical Engineering, University College Dublin, Ireland, 189 pp., 2009.

Neupert, J., Arnold, E., Schneider, K., and Sawodny, O.: Tracking and anti-sway control for boom cranes, Control Eng. Pract., 18, $31-44,2010$

O'Connor, W. J.: A Gantry Crane Problem Solved, J. Dyn. Syst.-T ASME, 125, 569-576, 2003.

O'Connor, W. J.: Wave-Based Analysis and Control of LumpModeled Flexible Robots, IEEE T. Robot., 23, 342-352, 2007.

O'Connor, W. J. and Fumagalli, A.: A Refined Wave-Based Control Applied to Nonlinear, Bending, and Slewing Flexible Systems, J. Appl. Mech., 76, 041005, doi:10.1115/1.3086434, 2009.

O'Connor, W. J. and McKeown, D. J.: A new approach to modal analysis of uniform chain systems, J. Sound Vib., 311, 623-632, 2008.

O’Connor, W. J., Ramos, F., McKeown, D. J., and Feliu, V.: Wavebased control of non-linear flexible mechanical systems, Nonlinear Dynam., 57, 113-123, 2009.

Sawodny, O., Aschemannb, H., and Lahres, S.: An automated gantry crane as a large workspace robot, Control Eng. Pract., 10, 1323-1338, 2002.

Schulze, T. and Chang, T. N.: Zero Vibration Position Control of a Spherical Pendulum for Control Systems Demonstration, American Control Conference, Marriott Waterfront, Baltimore, MD, USA, 738-743, July 2010.

Singhose, W. E. and Towel, S. T.: Double-Pendulum Gantry Crane Dynamics and Control, in: Proceedings of the 1998 IEEE International Conference on Control Applications Trieste, Italy, 1205-1209, September 1998.

Sun, N., Fang, Y., and Zhang, X.: Energy coupling output feedback control of 4-DOF underactuated cranes with saturated inputs, Automatica, 49, 1318-1325, 2013.

Tanaka, S. and Kouno, S.: Automatic measurement and control of the attitude of crane lifters; Lifter-attitude measurement and control, Control Eng. Pract., 6, 1099-1107, 1998.

Todd, M. D., Vohra, S. T., and Leban, F.: Dynamical measurements of ship crane load pendulation, in: Oceans 97 MTS/IEEE: Conference Proceedings, Hailfax, Canada, 2, 1230-1236, 1997. 
Vaughan, J., Karajgikar, A., and Singhose, W.: A Study of Crane Operator Performance Comparing PD-Control and Input Shaping, American Control Conference, San Francisco, CA, USA, 545-550, June 2011.

Yang, J. H. and Yang, K. S.: Adaptive Control for 3-D Overhead Crane Systems, in: Proceedings of the 2006 American Control Conference, Minneapolis, Minnesota, USA, 1832-1837, June 2006.
Zhong, B.: Load's 2-Degree of Freedom Swing Angle Model and Dynamic Simulation for Overhead Crane or Gantry Crane, Energy Procedia, 11, 1217-1223, 2011. 